\title{
RATIONAL APPROXIMATION NEAR ZERO SETS OF FUNCTIONS
}

\author{
PETER V. PARAMONOV
}

\begin{abstract}
The paper deals with the relation between global rational approximation and local approximation of the zero set. Also connections with the problem $f^{2} \in R(X) \Rightarrow f \in R(X)$ are studied.
\end{abstract}

The main result of this paper (Theorem 2 in $§ 2$ ) was obtained in Moskow and was discussed in some seminars and conferences in USSR. It was announced in the proceedings of the $1983 \mathrm{Kiev}$ conference on complex analysis, but no proof was provided. The other results were found while the author was visiting the CRM in Barcelona in the fall of 1987.

1. Let $X$ be a compact in $C$, and $R(X)$ the closure in $C(X)$ (with sup-norm) of the space of functions which are holomorphic on $X$.

For $f \in C(X)$ we will write $f \in R(X, x)$ for some $x \in X$, if there exists a neighbourhood $U$ of $x$ such that

$$
\left.f\right|_{\overline{X \cap U}} \in R(\overline{X \cap U}) .
$$

We let $N(f)$ stand for $\{x \in X \mid f(x)=0\}$ - the zero set of $f$.

We will consider the following two closely related problems.

Power problem (P-Pr). Is it true that for any function $f \in R(X)$ and $q>0$ the condition $f^{q} \in C(X)$ implies $f^{q} \in R(X)$ (same fixed branch)?.

Zero set problem (Z-Pr). Let $f \in C(X)$ and $f \in R(X, x)$ for all $x \in$ $X \backslash N(f)$.

Is it true that $f \in R(X)$ ?.

It is clear that these problems are trivial for $N(f)=\emptyset$, and that a positive answer to the Z-Pr implies the same answer to the P-Pr. Also it is not difficult to see, that P-Pr and Z-Pr are equivalent for $f$ in the class

$$
L(X) \equiv \mathrm{U}_{\rho>0} \operatorname{Lip}(\rho, X) .
$$

The author is grateful to the CRM for making possible his visit to Barcelona, and to the faculty of the Department of Mathematics of the UAB for many interesting discussions and remarks in seminars and private conversations. 
For the P-Pr the simpler case $q>1$ was considered in [5]. It was proved there that this problem has a positive answer for all $X$ and all $f \in L(X)$, but really the proof presented in [5] works only under the additional assumption $f^{q-1} \in L(X)$. One can find a simple example $(X \subseteq R)$, for which $f \in L(X)$, $q>1, f^{q}$ and $f^{q-1} \in C(X)$, but $f^{q}$ and $f^{q-1}$ are not in $L(X)$ (the branches are fixed and corresponding).

Given two compact sets $X$ and $Y, Y \subseteq X$ and $h(z) \in C(X)$. We will write

$$
h(z) \in L_{X}(Y)
$$

if there exist $\rho>0$ and a constant $c>0$, such that for every $z \in X$ and $w \in Y$ we have

$$
|h(z)-h(w)| \leq c|z-w|^{\rho} .
$$

The following Theorem has a proof absolutely like Theorem I in [5], except for a small change, wich will be described below.

Theorem 1. Let $f \in R(X), h \in L_{X}(N(f))$ and $h \in R(X, x)$ for all $x \in$ $X \backslash N(f)$. Then $f(z) \cdot h(z) \in R(X)$.

Corollary. Let $f \in R(X) \cap L_{X}(N(f))$, then for every $q>1, f^{q} \in R(X)$ is equivalent to $f^{q} \in C(X)$.

The corollary follows applying Theorem 1 to $h=f^{q-1}$.

To prove Theorem 1 we proceed as in [5] observing that we just need to worry about the squares (coming from the Vitushkin localization procedure) which intersect $N(f)$. To deal with these squares we use Lemma 3 in $[5$, p. 416] which turns out to be true under our weaker hypothesis. Concretely what we need is the following Lemma.

Lemma. Let $h$ be as in Theorem 1. There exiat $\rho=\rho(h)>0$ and $A=A(h)$, such that for every square $T_{\delta}$ with side length $\delta, T_{\delta} \cap N(f) \neq \emptyset$, and every function $g(z) \in C_{0}^{1}\left(T_{\delta}\right)$, we have

$$
\left|\int_{T_{6}} \int h(z) \frac{\partial g}{\partial z} d z d \bar{z}\right| \leq A \delta\|\nabla g\|_{\infty}\left(\delta^{p} \alpha\left(T_{\delta} \backslash X\right)+M^{1+\rho}\left(T_{\delta} \cap N(f)\right)\right)
$$

where $\alpha(\cdot)$ is $C$-analytic capacity and $M^{1+\rho}(\cdot)$ is the Hausdorff content of order $1+\rho($ see $[6]$, p. 1/45).

We close this section by stating an open problem dealing with $R^{\rho}(X)$, the closure in $\operatorname{Lip}(\rho, X)$ of the space of holomorphic functions on $X$.

Problem. Let $f \in R^{\rho}(X)$, and assume that $f^{q} \in \operatorname{lip}(\rho, X)$ for some $q>1$. Is it true that $f^{q} \in R^{p}(X)$ ? 
2. In $[3,4]$ we proved, that the $Z$ - $\operatorname{Pr}$ has a positive answer for all $X$ and all functions $f \in \operatorname{Lip}(\rho, X), \rho>\frac{1}{2}$. Now we are going to prove one theorem concerning this problem for all $\rho>0$, but for some special compact sets $X$. This result points in the direction that the Z-Pr has a positive answer also for all $X$ and all $f \in L(X)$. We need some notation.

Let $X$ be compact and $x \in X$. We say that $x$ is a point of stability (of the capacity of $C \backslash X)$ if one of the following two conditions holds.

i) There exists $\lim _{\delta \rightarrow 0} \log _{\delta}(\alpha(T(x, \delta) \backslash X)$ ), where $T(x, \delta)$ (here and below) is the square with center $x$ and side length $\delta$.

ii) $\lim _{\delta \rightarrow 0} \inf \log _{\delta}(\alpha(T(x, \delta) \backslash X)) \geq 2$. If we denote the $\lim$ in (i) by $\lambda(x)$, then (i) is equivalent to

$$
\alpha(T(x, \delta) \backslash X)=\delta^{\lambda(x)+o(1)} \text { as } \delta \longrightarrow 0,
$$

and (ii) is the same that

$$
\alpha(T(x, \delta) \backslash X)=0\left(\delta^{2-\varepsilon}\right) \text { for any fixed } \varepsilon>0 \text { as } \delta \longrightarrow 0 .
$$

Let now $X^{*}=\{x \in X \mid x$ is unstable $\}$.

Theorem 2. If $X^{*}=$ then the Z.Pr has a positive answer for all $f \in L(X)$.

Corollary 1. Let $f \in L(X)$ and assume $f \in R(X, x)$ for all $x \in X \backslash N(f)$. Then $f \in R(X, x)$ also for all $x \in X \backslash\left(N(f) \cap \bar{X}^{*}\right)$.

The proof of Theorem 2 and the main idea in the proof of Lemma $2[5]$ allow to get also the following corollary:

Corollary 2. Let $f \in \operatorname{Lip}(\rho, X), \rho>0$, and assume $f \in R(X, x)$ for all $x \in X \backslash N(f)$. If

$$
M^{1 /(1-p+\varepsilon)}\left(N(f) \cap \bar{X}^{*}\right)=0 \text { for some } \varepsilon>0 \text {, then } f \in R(X) .
$$

We remark that for $\rho>\frac{1}{2}$ the last hypothesis is automatically satisfied and so we obtain the main result of $[3,4]$.

Some preliminary results and remarks. For $T_{\delta}=T(z, \delta)$ we will write $r \cdot T_{\delta}=T(z, r \cdot \delta)$, for each $r>1$. As in [4] we will need the following

Theorem 0 . Let $T$ be a square and $0<\tau \leq 1$. If $f \in C(4 T),\|f\|_{4 T} \leq 1$ and $f$ is analytic on $4 T^{0} \backslash M_{c}(f)$, where $M_{c}(f)=\{z \in 4 T|| f(z) \mid \leq \varepsilon\}$, then

$$
\left|\int_{\partial T} f(z) d z\right| \leq C(\tau) \cdot \varepsilon^{1-\tau} \cdot \alpha\left(M_{\varepsilon}(f) \cap T\right) .
$$


Remark 1. Assume $\mathcal{D}$ is Jordan domain with rectifiable boundary $\partial \mathcal{D}$, and $G$ any open neighbourhood of $\overline{\mathcal{D}}$. If the estimate of the integral due to Melnikov and Vitushkin $([6]$, p. 158$)$ is satisfied in $\mathcal{D}$, then it is easily seen that we also have

$$
\left|\int_{\partial \mathcal{D}} f(z) d z\right| \leq C(\mathcal{D}, G, \tau) \cdot \varepsilon^{1-\tau} \cdot\|f\|_{G}^{\tau} \alpha\left(M_{\varepsilon}(f) \cap \mathcal{D}\right),
$$

where $M_{e}(f)=\{z \in G|| f(z) \mid \leq \varepsilon\}, \quad f \in C(\bar{G})$ and $f$ is analytic on $G \backslash M_{\varepsilon}(f)$. Problem. Is estimate (2) true if we take $G=\mathcal{D}$ ?.

In applications to rational approximation we really don't need the condition $G=\mathcal{D}$, but it seems useful to have (2) with $\tau=0$.

As it turns out estimates (2) and (1) are not true for $\tau=0$. We present an example here.

Let $S$ be a compact with $m(S)>0$ (where $m(\cdot)$ is plane Lebesgue measure), with empty interior and with connected complement. Set $\varphi(z)=\int_{S} \frac{d m(\zeta)}{z-\zeta}$, so that $\varphi \in C(\bar{C}), \quad \varphi(\infty)=0$, and $\varphi$ is analytic on $\bar{C} \backslash S$. Let now $T$ be a square, containing $S$. Then

$$
\int_{\partial T} \varphi(z) d z=2 \pi i \lim _{z \rightarrow \infty} z \varphi(z)=2 \pi i m(S) \neq 0 .
$$

By the Mergelian's Theorem [2] for any $\varepsilon>0$ there exists polynomial $P(z)$ such that $\|\varphi(z)-P(z)\|_{s} \leq \varepsilon$.

Applying (2) with $f=\varphi-P, \mathcal{D}=T, G=4 T$ (and $\tau=0$ ) we would get

$$
2 \pi m(S)=\left|\int_{\partial T} f(z) d z\right| \leq C \cdot \varepsilon,
$$

which is a contradiction for $\varepsilon$ small enough.

We will use also two theorems of Vitushkin ([6], p. 158; for Theorem V1 see $[3]$, p. 104).

Theorem V1. Let $f \in C(C)$. Then $f \in R(X)$ provided there exist $r \geq 1$ and $a(\delta) \longrightarrow 0$ as $\delta \longrightarrow 0$, such that for any square $T_{\delta}$ of side length $\delta$ we have

$$
\left|\int_{\partial T_{\delta}} f(z) d z\right| \leq \alpha(\delta)\left[\alpha\left(r T_{\delta} \backslash X\right)+\delta^{2}\right] .
$$

Conversely if $f \in R(X)$ we obtain (g) with $a(\delta)=c \omega(f, \delta), r=1$ and without $\delta^{2}$ in the right hand-side.

Theorem V2. Let $E$ be a bounded set with $\alpha(E)=\alpha>0$, and $\left\{E_{j}\right\}_{j=1}^{N}$ a finite number of sets $E_{j} \subseteq E$ such that any square $T_{\alpha}$ with side $\alpha$ intersects at most $p$ ( $p \geq 1$ is a fixed integer) $E_{j}$ 's. Then for some absolute constant $c$ 


$$
\sum_{j=1}^{N} \alpha\left(E_{j}\right) \leq c \cdot p \cdot \alpha(E)
$$

Proof of Theorem 2: Now we fix a compact $X$ with $X^{*}=0$, a function $f \in \operatorname{Lip}(\rho, X), \rho>0$, and let us suppose, that $f \notin R(X)$. After several lemmas we will have a contradiction.

We will denote by $C$ an absolute constant and by $A$ a constant depending only on $f$ (on $\rho$ ).

Both of them may vary from an inequality to another.

Let $T_{\delta}$ be any square with side $\delta$ and put $\alpha\left(5 T_{\delta} \backslash X\right) \equiv 5 \cdot \delta^{\beta}$. Then $\beta>1$.

Take $\delta_{1}=\delta^{\beta}$ and consider a non-overlapping family $\left\{T_{j}\right\}$ of squares of side $\delta_{1}$ and centers $\left\{z_{j}\right\}$, covering the plane. Let $\varphi_{j} \in C_{0}^{1}\left(2 T_{j}\right), \quad\left|\nabla \varphi_{j}\right| \leq C \delta_{1}^{-1}$ and $\sum_{j} \varphi_{j} \equiv 1$ on $C$. We denote by $j^{t}$ the indexes $j$ for which $\left.f\right|_{3 T_{j}} \in R\left(X \cap 3 T_{j}\right)$ and $3 T_{j} \subset 5 T_{\delta}$.

Lemma 1. Let $t$ be a point in $4 T_{\delta}$ such that

$$
\alpha\left(\left(T(t, \varepsilon) \cap 5 T_{\delta}\right) \backslash X\right) \leq C \cdot \varepsilon^{\frac{1}{1-\nu}}
$$

for some, $\nu, \quad 0 \leq \nu<1$, and all $\varepsilon, \quad \delta_{1} \leq \varepsilon \leq 10 \delta$.

Then we have

$$
\sum_{j^{\prime}: t \notin 3 T_{j^{\prime}}} \frac{\alpha\left(2 T_{j^{\prime}} \backslash X\right)}{\left|t-z_{j^{\prime}}\right|} \leq C \delta_{1}^{\nu}
$$

Proof: Inequality (4) will follow from Theorem V2 and the following elementary result.

Sublemma. Let $0<a_{1} \leq a_{2}, \ldots$, and $\alpha_{1}<a$. If a sequence $\left\{\alpha_{n}, \alpha_{n} \geq 0\right\}$ satisfies the conditions $\sum_{p=1}^{m} \alpha_{p} \leq a_{m}$ for all $m \geq 1$ and $\sum_{p=1}^{\infty} \alpha_{p} \leq a$, then

$$
\sum_{p=1}^{\infty} \frac{\alpha_{p}}{p} \leq \sum_{m=1}^{M} \frac{a_{m}}{m^{2}}+\frac{a}{M}
$$

where $M$ is defined by $a_{M} \leq a<a_{M+1}$.

In particular, if $a_{m} \leq a$ for all $m \geq 1$, then

$$
\sum_{p=1}^{\infty} \frac{\alpha_{p}}{p} \leq \sum_{m=1}^{\infty} \frac{a_{m}}{m^{2}}
$$


Proof: The sum $\sum_{p=1}^{\infty} \frac{\alpha_{p}}{p}$ is maximized by

$$
\alpha_{p}= \begin{cases}a_{p}-a_{p-1} & \text { for } p \leq M \text { and } a_{0}=0 \\ a-a_{M} & \text { for } p=M+1 \\ 0 & \text { for } p>M+1\end{cases}
$$

A computation now gives the sublema. To prove Lemma 1 let $\left.\mathcal{D}_{p}=T\left(t,(p+4) \delta_{1}\right) \backslash T\left(t, p \delta_{1}\right)\right), \quad p \geq 1$, and $\alpha_{p}=\alpha\left(\mathcal{D}_{p} \cap 5 T_{\delta} \backslash X\right) / \delta_{1}$. By Theorem V2

$$
\sum_{j^{\prime}: 2 T_{j^{\prime}} \subseteq \mathcal{D}_{p}} \frac{\alpha\left(2 T_{j^{\prime}} \backslash X\right)}{\left|t-z_{j^{\prime}}\right|} \leq \frac{C_{1} \alpha_{p}}{p} .
$$

Now we use the sublemma with $a=C_{1}$ and $\left.a_{m}=C_{1} \delta_{1}^{-1}[(m+4)] \delta_{1}\right]^{\frac{1}{1-v}}$. Lemma 1 is proven.

We will construct by induction squares $S_{n}$ such that $f \notin R\left(X_{n}\right)$, where $X_{n}=X \cap S_{n}$, and for any square $T_{\delta}$ we will have

$$
\left|\int_{\partial T_{\delta}} f d z\right| \leq a(\delta) \cdot\left[\alpha\left(5 T_{\delta} \backslash X_{n}\right)+\delta^{\frac{b}{1-\theta_{n}}}\right]
$$

with $a(\delta) \longrightarrow 0$ as $\delta \longrightarrow 0$, and $\theta_{n} \geq \theta_{1}+\frac{n-1}{2} \rho, \quad \theta_{1}<\frac{1}{2}$.

For some large $n: \frac{1}{1-\theta_{n}} \geq 2$, and so Theorem VI will imply $f \in R\left(X_{n}\right)$, which is the desired contradiction.

For $n=1$ we let $S_{1}$ be any square such that $f \notin R\left(X_{1}\right)$, where $X_{1}=S_{1} \cap X$. For any square $T_{\delta}$ we have (since $f \in \operatorname{Lip}(\rho, X)$ )

$$
\left|\int_{\partial T_{b}} f d z\right| \leq A \delta^{1+\rho}=a(\delta) \delta^{1+\rho / 2}=a(\delta) \cdot \delta^{\frac{2}{1-\theta_{1}}}
$$

where $\theta_{1}=\frac{\rho / 2}{i+\rho / 2}$. So (5) is satisfied for $n=1$.

For the inductive step we need two lemmas.

Lemma 2. Let $Q_{1}$ be a square and

$$
f \notin R\left(X \cap Q_{1}\right) \equiv R\left(Y_{1}\right), \text { where } Y_{1}=X \cap Q_{1}
$$

Suppose, that there exist some $\theta, 0<\theta<1$, and $a_{1}(\delta) \longrightarrow 0$ as $\delta \longrightarrow 0$ such that for any square $T_{\delta}$ we have (5) with $X_{n}=Y_{1}$ and $\theta_{n}=\theta$. Fix any $\theta^{\prime}$, $0<\theta^{\prime}<\theta$.

Then there exists a square $Q_{2} \subseteq Q_{1}$ such that $\left.f\right|_{Y_{2}} \notin R\left(Y_{2}\right)$, where $Y_{2}$ $=Y_{1} \cap Q_{2}=X \cap Q_{2}$, and for every square $T_{\varepsilon} \subseteq Q_{2}$, satisfying the condition

$$
\alpha\left(T_{e} \backslash Y_{2}\right) \geq \varepsilon^{\frac{1}{1-\theta^{\prime}}}
$$


we have $f \in R\left(T_{\varepsilon} \cap Y_{2}\right)$

Proof: If for some square $T_{\delta}$ inequality (3) is not true (for $Y_{1}$ instead of $X$ ), then using (5) with $X_{n}=Y_{1}$ and $\theta_{n}=\theta$, we get

$$
\alpha\left(T_{\delta} \backslash Y_{1}\right) \leq \delta^{\frac{1}{2-\delta}}
$$

But if Lemma 2 is not true, then, using (6) and (7), by induction, we will find a point $t \in Y_{1}$ such that

$\liminf _{\delta \rightarrow \infty}\left(\log _{\delta}\left(\alpha\left(T(t, \delta) \backslash Y_{1}\right)\right) \leq \frac{1}{1-\theta^{\prime}}<\frac{1}{1-\theta} \leq \limsup _{\delta \rightarrow \infty}\left(\log _{\delta}\left(\alpha\left(T(t, \delta) \backslash Y_{1}\right)\right)\right)\right.$

As $\theta$ in (5) is less than $\frac{1}{2}$ (otherwise $f \in R\left(Y_{1}\right)$ by Theorem V1), we have $t \in Y_{I}^{*} \subseteq X^{*} \neq \emptyset$, contradincting our assumption $X^{*}=\emptyset$.

Lemma 3. Let $Q_{2}$ be the square in the statement of Lemma 2, e.g.

(a) $f\left\{_{Y_{3}} \notin R\left(Y_{2}\right)\right.$, where $Y_{2}=X \cap Q_{2}$.

(b) For some $0<\theta<1$ we have for any square $T_{\delta}$ :

$$
\left|\int_{\partial T_{b}} f(z) d z\right| \leq \alpha(\delta)\left[\alpha\left(5 T_{\delta} \backslash Y_{2}\right)+\delta^{\frac{1}{1-z}}\right]
$$

(c) Any fixed $\theta^{\prime}, \quad 0<\theta^{\prime}<\theta$, has the following property: for any square $T_{\epsilon} \subseteq Q_{2}$ and satisfying (6) we have $f \in R\left(T_{\epsilon} \cap Y_{2}\right)$. Then for any fized $\tau$, $0<\tau<1$, there exist $a_{1}(\delta) \longrightarrow 0$ as $\delta \longrightarrow 0$ such that for any square $T_{\delta}$ :

$$
\left|\int_{a T_{6}} f(z) d z\right| \leq a_{1}(\delta)\left[\alpha\left(5 T \backslash Y_{2}\right)+\delta^{\frac{1}{1-\left(\alpha+\sigma^{\prime}\right)(1-\tau)}}\right]
$$

Remark. The meaning of Lemma 3 is that (5) holds with $X_{n}$ replaced by $Y_{2}$ and $\theta_{n}$ by $\theta_{(2)}=\left(\theta^{\prime}+\rho\right)(1-\tau) \geq \theta+\rho / 2$ for $\tau$ and $\left(\theta-\theta^{\prime}\right)$ small enough. Before going on the proof of Lemma 3 we complete the proof of Theorem 2 . By induction we find compacts $X_{n}$ with $f \notin R\left(X_{n}\right), X_{n}=X \cap S_{n}$ (put $S_{n-1}=Q_{1}$ and $S_{n}=Q_{2}$ in Lemmas 2 and 3 ), for which (5) holds with $\theta_{n}=\theta_{1}+\frac{n-1}{2} \rho$. When $\frac{1}{2}<\theta_{n}<1$ we have a contradiction.

Proof of Lemma 3: Fix any square $T_{\delta}$ with side length $\delta$, assuming also that $T_{\delta}$ is diadic [1]. The cases $5 T_{\delta} \nsubseteq Q_{2}$ or $\alpha\left(5 T_{\delta} \backslash Y_{2}\right) \geq \delta^{\frac{1}{1-e^{\prime}}}$ are trivial, so we will consider the case

$$
5 T_{\delta} \subseteq Q_{2} \quad \text { and } \quad \alpha\left(5 T_{\delta} \backslash Y_{2}\right) \equiv 5 \delta^{\beta}<\delta^{\frac{1}{1-\theta^{\gamma}}}
$$

So we have $5 \delta^{\beta}<<\delta$ if $\delta$ is small enough. 
Now we divide $5 T_{\delta}$ into equal diadic squares $\left\{T\left(j_{1}\right)\right\}$ with side length $\delta_{1}$, $\frac{1}{2} \delta^{\beta} \leq \delta_{1}<\delta^{\beta}$. We call a square $T\left(j_{1}\right)$ "good" and write $j_{1}=j_{1}^{\prime}$ if $T\left(j_{1}\right)$ satisfies the following condition (for $n=1, J_{n}^{\prime}=j_{1}^{\prime}$ ):

$$
\left.f\right|_{4 T\left(J_{n}^{\prime}\right)} \in R\left(4 T\left(J_{n}^{\prime}\right) \cap Y_{2}\right) \text {. }
$$

For the remaining "bad" squares we use the notation $T\left(j_{n}^{\prime \prime}\right)$. We will continue our procedure only with "bad" squares, for which (6) gives

$$
\alpha\left(4 T\left(j_{1}^{\prime \prime}\right) \backslash Y_{2}\right) \leq\left(4 \delta_{1}\right)^{\frac{1}{1-\theta^{\prime}}} \leq 16 \delta^{\frac{1}{1-\theta^{\prime}}}
$$

Assume that the squares $\left\{T\left(j_{1}^{\prime \prime}, \ldots, j_{n-1}^{\prime \prime}, j_{n}\right\}\right.$ have been constructed. For short we put

$$
\left(j_{1}^{\prime \prime}, \ldots, j_{n-1}^{\prime \prime}, j_{n}\right)=\left(J_{n}\right) \text {. }
$$

For the "good" squares $T\left(J_{n}\right)$, which satisfy (8) we use $T\left(J_{n}^{\prime}\right)$ and they will not be divided again. Every "bad" square $T\left(J_{n}^{\prime \prime}\right)$ will divided into equal diadic squares $\left\{T\left(J_{n}^{\prime \prime}, j_{n+1}\right)\right\} \equiv\left\{T\left(J_{n+1}\right)\right\}$ with centers $\left\{z_{J_{n+2}}\right\}$ and side lenght $\delta_{n+1}$ satisfying

$$
\frac{1}{2} \delta^{\frac{1}{2-\theta^{\prime}}} \leq \delta_{n+1}<\delta_{n}^{\frac{1}{1-\theta^{\prime}}}
$$

Using (6) we find that, for every $2 T\left(J_{n}^{\prime \prime}\right)$,

$$
\alpha\left(2 T\left(J_{n}^{\prime \prime}\right) \backslash X\right) \leq \alpha\left(4 T\left(J_{n}^{\prime \prime}\right) \backslash X\right) \leq\left(4 \delta_{n}\right)^{\frac{s}{2-\theta^{\prime}}} \leq 16 \delta_{n+1} .
$$

We stop this procedure when $\delta_{N} \leq\left(\delta^{\beta}\right)^{\frac{1}{\beta}}<\delta_{N-1}$.

Evidently, as $\delta_{n}<\left(\delta^{\beta}\right)^{\frac{n-1}{1-\theta^{\prime \prime}}}$, we have

$$
N \leq 1+\frac{1-\theta^{t}}{\rho} \leq \frac{2}{\rho} .
$$

Then

$$
5 T_{\delta}=\left[\cup_{n=1}^{N} \cup_{\left(J_{n}^{\prime}\right)} T\left(J_{n}^{\prime}\right)\right] \cup\left[\cup_{\left(J_{N}^{\prime \prime}\right)} T\left(J_{n}^{\prime \prime}\right)\right] .
$$

By a lemma of Harvey and Polking ([1], p. 43) there exists a partition of unity $\left\{\varphi_{J_{n}^{\prime}}\right\}_{n=1}^{N} \cup\left\{\varphi_{J_{N}^{\prime \prime}}\right\}$ with $\operatorname{Supp} \varphi_{J_{n}} \subseteq 2 T\left(J_{n}\right), \quad\left|\nabla \varphi_{J_{n}}\right| \leq \frac{c}{\delta_{n}}$ and

$$
\sum_{n=1}^{N} \sum_{\left(J_{n}^{\prime}\right)} \varphi J_{n}^{\prime}+\sum_{\left(J_{N}^{\prime \prime}\right)} \varphi_{J_{N}^{\prime \prime}} \equiv 1 \text { on } 4 T_{\delta} .
$$

Let $f_{J_{n}}(z) \equiv \frac{1}{2 \pi i} \iint \frac{f(\zeta)-f(z)}{\zeta-z} \frac{\partial \varphi_{J_{n}}(\zeta)}{\partial \bar{\zeta}} d \vec{\zeta} \wedge d \zeta$. By Vitushkin Theorem ([6], p. 148) we have $f_{J_{n}^{\prime}} \in R\left(Y_{2}\right), \quad\left\|f_{J_{n}}\right\|_{\infty} \leq c \delta_{n}^{\rho}$, and for $t \notin 3 T_{\left(J_{n}^{\prime}\right)}$

$$
\left|f_{J_{n}^{\prime}}(t)\right| \leq \frac{c \delta_{n}^{\rho} \alpha\left(2 T\left(J_{n}^{\prime}\right) \backslash X\right)}{\left|t-z_{J_{n}^{\prime}}\right|}
$$


Consider the function

$$
\begin{aligned}
& G(z)=\sum_{n=1}^{N} \sum_{\left(J_{n}^{\prime}\right)} f_{J_{n}^{\prime}}(z) \in R(X), \quad \text { and } \\
& F(z)=f(z)-G(z) .
\end{aligned}
$$

Let $\Omega$ be a set of nonanalyticity of $F$ in $4 T_{6}$, then $\Omega$ belong to $4 \delta_{N}-$ neighbourhood of $N(f)([6]$, p. 148).

For every $t \in \Omega$ and $\delta_{n}<\varepsilon<10 \delta$ we have by (6) and (9):

$$
\alpha\left(T(t, \varepsilon) \cap 5 T_{\delta} \backslash Y_{2}\right) \leq c \varepsilon^{\frac{1}{1-\theta^{\prime}}}
$$

and $t \notin 3 T\left(J_{n}^{t}\right)$ for $n<N$.

To estimate $G(z)$ on $\Omega$ we use Lernma 1 (with $\nu=0$ for $n=N$ and $\nu=\theta^{\prime}$ for all $1 \leq n \leq N-1$ ), Theorem V2 (see also (9)), and we recall that the induction procedure is finite $\left(N \leq \frac{2}{p}\right)$.

We get $\| G \mid \xi_{4 T_{6}} \leq A \delta^{\rho}$ and

$$
\|G\|_{\Omega} \leq c \delta_{N}^{\rho}+\sum_{n=1}^{N} A_{n} \delta_{n}^{\rho} \cdot \delta_{1}^{\theta^{\prime}} \leq A\left(\delta^{\beta}\right)^{\rho+\theta^{\prime}}
$$

Since $\|f\|_{\Omega} \leq A\left(\delta_{n}\right)^{\rho} \leq A \delta^{\beta} \leq A\left(\delta^{\beta}\right)^{\rho+\theta^{\prime}}$, (the case $\rho=\frac{1}{2}$ or $\theta^{\prime}>\frac{1}{2}$ is evident), then

$$
\|F\|_{\Omega} \leq A \delta^{\beta\left\{\rho+\theta^{\prime}\right)} .
$$

By Theorem V1 (as $G(z) \in R\left(Y_{2}\right)$ ), we have

$$
\left|\int_{\partial T_{\delta}} G d z\right| \leq A \delta^{\rho} \alpha\left(T_{\delta} \backslash Y_{2}\right) \leq A \delta^{\rho} \cdot \delta^{\beta}
$$

and by Theorem 0 (for $\left.\tau_{1}=\tau / 2\right)$ we have $\left|\int_{\partial T_{b}} F d z\right| \leq A(\tau, \rho)\left[\delta^{\beta\left(\rho+\theta^{\prime}\right)}\right]^{1-\frac{\tau}{2}} \cdot \delta$. Hence

$$
\left\{\int_{\partial T_{6}} f d z \mid \leq a_{1}(\delta)\left[\delta^{\beta}+\delta^{\beta\left(\rho+\theta^{\prime}\right)(1-\tau)+1}\right]\right.
$$

and $a_{1}(\delta) \longrightarrow 0$ as $\delta \longrightarrow 0$.

Finally, if $\beta \leq \beta\left(\rho+\theta^{\prime}\right)(1-\tau)+1$ then (5) holds, but if $\beta>\beta\left(\rho+\theta^{\prime}\right)(1-\tau)+1$, that is, $\beta>1 /\left[1+\left(\rho+\theta^{\prime}\right)(1-\tau)\right]$, we also have $\left(5^{\prime}\right)$ : Lemma 3 and Theorem 2 are proved. 


\section{References}

1. R. HARVEY AND J. POLKING, Removable singularities of solutions of partial differentail equations, Acta Math. 125 (1970), 39-56.

2. S.N. MERGELIAN, Uniform approximations of functions of a complex variable; Uspekhi Mat. Nauk Amer. Math. Soc. Translation 101 M.R. 15 (1954), 612 .

3. P.V. PARAMONOV, On the interconnection of local and global approximations by holomorphic functions, Math. USSR Isv. 20 (1983), 103-118.

4. P.V. PARAMONOV, On a sufficient condition for approximability of a function by rational fractions, Soviet Math. Dokl. 27 (1) (1983).

5. P.V. PARAMonov, O vozmojnosti delenia i vozvedenia v drobnuiu stepen v algebre ratsionalnih funckci., Izvestia Akademii Nauk SSSR 51 (2) (1987), Russian.

6. A.G. VitushKin, Analytic capacity of sets in problems of approximation theory, Russian Math. Surveys 22 (1967), 139-200.

Engineering Institute Department of Mathematics ul. Krasnokazarmennaia N. 17

Moskow Power

Moskow USSR

Rebut el 26 d'abril de 1988 